\title{
Faecal Microbiota Transplantation is Effective for the Initial Treatment of Clostridium difficile Infection: A Retrospective Clinical Review
}

\author{
Niloufar Roshan · Annabel K. Clancy · Thomas J. Borody
}

Received: July 1, 2020 / Accepted: September 3, 2020 / Published online: September 26, 2020

(C) The Author(s) 2020

\begin{abstract}
Introduction: Clostridium difficile (C. difficile) infection (CDI) is commonly recognised as a nosocomial infection but is increasingly identified in patients in the community. Antimicrobial exposure which compromises gut microbiota is the main risk factor for CDI, although antibiotics remain the main treatment for this infection. Faecal microbiota transplantation (FMT) is also an effective treatment for CDI. FMT involves the transfer of microbiota from a healthy donor to an unwell patient. Currently FMT is mostly used after repeated antibiotic treatments fail to cure CDI. This study investigated the effect of FMT as first-line treatment for CDI to avoid repeated antibiotic damage of the microbiome.
\end{abstract}

Methods: This retrospective, single-centre study included 59 patients between 2012 and 2017 whose first episode of CDI was treated with FMT. The patients' symptoms and presence of $C$. difficile in stool samples both at the baseline and post treatment were documented. Results: Fifty-four patients completed a final stool test 4-8 weeks post treatment in which $98 \%$ of patients were negative for C. difficile. There were no adverse effects. There was a significant reduction in abdominal pain, diarrhoea, bloating and blood in the stool at 4-8 weeks post treatment. Data from 24 patients who completed an extended 6 months followup showed significant reduction in abdominal pain, diarrhoea and blood in the stool.

Conclusion: This study demonstrates the safety and efficacy of FMT as first-line treatment for patients' initial episode of CDI. Future randomised studies are required to confirm FMT as the initial treatment for CDI.

Keywords: Clostridium difficile; Faecal microbiota transplantation; Gut microbiota

Digital Features To view digital features for this article go to https://doi.org/10.6084/m9.figshare.12907031

N. Roshan · A. K. Clancy · T. J. Borody $(\bowtie)$

Centre for Digestive Diseases, Five Dock, NSW, Australia

e-mail: thomas.borody@cdd.com.au 


\section{Key Summary Points}

Why carry out the study?

In the USA, the Food and Drug Administration (FDA) mandates that CDI can only be treated with FMT after two treatment failures with antibiotics

Given CDI often follows antibiotic use, and the high efficacy of FMT in the treatment of recurrent CDI it makes sense to treat with FMT early, to eradicate CDI

This study aimed to investigate the safety, efficacy and clinical outcomes in patients with a first episode of CDI treated with FMT as first-line therapy

\section{What was learned from the study?}

First-line treatment with FMT demonstrated high efficacy $(98 \%$ eradication), low rates of recurrence, resolution of symptoms and no adverse events

This study demonstrates the efficacy and safety of FMT treatment for patients with a first episode of CDI

Our study supports the use of FMT as an optimal first-line treatment of CDI rather than waiting for multiple recurrences

\section{DIGITAL FEATURES}

This article is published with digital features to facilitate understanding of the article. To view digital features for this article go to https://doi. org/10.6084/m9.figshare.12907031.

\section{INTRODUCTION}

Clostridium difficile (C. difficile) is an anaerobic, Gram-positive, spore-forming bacillus that can cause gastrointestinal disease in humans [1].

The clinical symptoms arising from $C$. difficile infection (CDI) range from mild or self-limiting diarrhoea to severe diarrhoea and sequelae such as toxic megacolon or fulminant colitis [2]. CDI is a public threat in both the healthcare and community settings. It is a major cause of healthcare-associated diarrhoea in all areas of the world especially in the northern hemisphere [1]. The ingestion of $C$. difficile organism does not necessarily cause infection, since the colonic microbiota resist the colonisation and overgrowth of C.difficile. CDI usually follows gut microbiota disruption post antimicrobial exposure, leading to proliferation and germination of $C$. difficile spores into vegetative cells and results in toxin production in the intestine [3].

Several studies have shown that antimicrobial exposure has been the main contributing risk factor for CDI. Hospitalised elderly patients receiving antimicrobial therapy remain the high-risk group; however, CDI is increasingly observed in younger populations with no exposure to antimicrobials or healthcare settings [4]. Another group at high risk of acquiring CDI are those who have inflammatory bowel disease (IBD) such as Crohn's disease or colitis [5].

CDI is a major public health problem associated with the emergence of antibiotic-resistant bacteria. According to the American and European surveillance protocol of CDI, 500,000 infections were reported in the USA in 2011 [6] and 7711 cases were reported in Europe in 2016 [7]. One of the major issues in the management of CDI is the recurrence of infection, which is estimated to be 33\% after a first episode of CDI, and that complicates the therapeutic approaches. The relapse rate after treatment with metronidazole and vancomycin is estimated to be $50 \%$ and $37 \%$, respectively [8]. According to a study by Lessa et al. [9], among cases of community-acquired CDI, in the USA the recurrence rate and death within 30 days of diagnosis were estimated to be $14 \%$ and $1 \%$, respectively, while higher rates of recurrence (21\%) and death (9\%) were reported among patients with healthcare-associated infection [9]. Guidelines from the Infectious Diseases Society of America (IDSA) and Society for 
Healthcare Epidemiology of America (SHEA) in 2017 list that standard care for severe CDI includes intravenous administration of metronidazole and oral administration of vancomycin; for those not responding to these antibiotics, alternative treatments such as tigecycline and intravenously administered immunoglobulin can be used. Colectomy is also recommended for fulminant cases of CDI. However, after completing a course of antibiotics, $10-30 \%$ of the patients usually experience return of symptoms and recurrence of the disease. This risk increases to $60 \%$ after a third episode [6]. Overall, the increasing prevalence of hypervirulent $C$. difficile strains, the antimicrobial treatment failure, patients with severe complications and cases with multiple recurrences have driven the search for alternative and new treatment options.

Faecal microbiota transplantation (FMT) involves the infusion of healthy human faecal flora into the bowel of an unwell recipient, with an apparent success rate of approximately 90\% for relapsing CDI $[4,10]$. Since antibiotics can further deplete healthy microbiota, initial treatment of CDI with FMT may avoid this damage to the microbiome. FMT from a healthy donor counteracts the infection susceptibility by recovering the natural microbiota in the patients. However, in the USA, the Food and Drug Administration (FDA) mandates that CDI can only be treated with FMT after two treatment failures with antibiotics [10]. Subsequently, there have been limited studies on the use of FMT as the first line of treatment for CDI. Previously a small proof of concept trial investigated the effect of FMT for primary CDI, which suggested FMT as an alternative to antibiotic therapy in treating primary CDI [11]. Our group has also previously reported preliminary results demonstrating efficacy [12].

Given that CDI often follows antibiotic use, which may result in depleted bacterial classes, it appears illogical to continue to use further antibiotics to treat CDI. Conversely, given the high efficacy of FMT, it makes sense to treat with FMT early, eradicate CDI, and repair microbiome damage and replace missing flora components simultaneously. Therefore, this study aimed to investigate the safety, efficacy and clinical outcomes in patients with a first episode of CDI treated with FMT as first-line therapy. This study also aimed to provide evidence to modify and improve current treatment and clinical practice.

\section{METHODS}

This is a retrospective, single-centre review of patients with a first occurrence of CDI treated with FMT as first-line treatment. Patients 18 years or older with first episode of CDI treated with FMT between January 2012 and December 2017 were identified from the internal medical records database. The standard treatment protocol during this time period consisted of a 10-14 day course of antibiotics such as vancomycin and/or metronidazole followed by two FMT infusions. The initial infusion was administrated via colonoscopy, followed the next day by rectal enema. The regulation, production and use of fresh FMT were performed in accordance with recommendations at the time of treatment [13-16]. Donors were regularly screened and both their stool and blood were routinely tested for any pathology. Stool was homogenised with sterile saline and filtered. The patients in this study received FMT from different donors that were actively donating at the centre within this time period.

A review of medical records was conducted in November 2019. Patients' symptoms, before FMT, at 4-8 weeks and then 6 months after FMT were collated. Stool test results (culture and toxin assay) were recorded at baseline and 4-8 weeks post treatment. Treatment success was defined as negative stool culture and toxin assay. Further information on patient demographic data and clinical data such as age, gender, pathology results, symptoms, treatments, number of FMT infusions, pre-treatment antibiotics and adverse events were collated. The primary outcome was defined as negative stool culture and toxin assay at 8 weeks post treatment. The secondary outcomes included change in symptoms at 8 weeks and 6 months post treatment. 
Statistical analysis was conducted using GraphPad Prism v.8 (La Jolla, CA, USA) software. Descriptive statistics were conducted and statistical differences between three or more sets of data were analysed using one-way analysis of variance and nonparametric technique, followed by Tukey's multiple comparison post test if the $P$ value was significant. Wilcoxon matched-pairs signed rank test was used to compare the differences between two sets of data. $P$ values less than 0.05 were considered significant. This study has been approved by the Centre for Digestive Diseases Human Research Ethics Committee (CDD19/C06). A waiver of consent

Table 1 Baseline demographic data

\begin{tabular}{ll}
\hline Parameter & Value \\
\hline Gender $(N)$ & $\mathrm{F}(48) / \mathrm{M}(11)$ \\
Age, median (years) & 47 \\
Pre-treatment abx for CDI & $1(1.7 \%)$ \\
No abx & $35(59.3 \%)$ \\
Only van & $2(3.4 \%)$ \\
Only mtz & $11(18.6 \%)$ \\
Van + mtz & $10(17 \%)$ \\
Van + other abx & $C$. difficile, N (\%) \\
Pre-treatment pathology testing for & $16(27.1 \%)$ \\
PCR & $14(23.7 \%)$ \\
Culture & $29(49.2 \%)$ \\
PCR + culture & \\
Symptoms, $N$ & 41 \\
Abdominal pain & 15 \\
Bleeding & 22 \\
Bloating & 22 \\
Diarrhoea & 14 \\
Flatulence & 12 \\
Vomiting/nausea &
\end{tabular}

$V a n$ vancomycin, $m t z$ metronidazole, $a b x$ antibiotic, $P C R$ polymerase chain reaction for $C$. diffcile toxin was granted for this study. This study was performed in accordance with the Helsinki Declaration of 1964.

\section{RESULTS}

Fifty-nine patients (11 men) with a median age of 47 years were eligible for inclusion in the study. Out of 59 patients, $44 \%$ had other medical conditions with $20 \%$ having a history of gastrointestinal disorders such as IBD and irritable bowel syndrome (IBS). All 59 patients tested positive for $C$. difficile toxin on PCR and/ or had a positive culture or both. Some $41 \%$ of patients had three or more of the symptoms listed in Table 1 . The most common symptoms were abdominal pain, diarrhoea and bloating. Fifty-eight patients had pre-treatment antibiotics for CDI (Table 1). Fifty-four patients completed the pre- and post-stool test and symptom questionnaire and were included in the posttreatment analysis.

Fifty-four patients completed a final stool test at a median 5 weeks ( $4-8$ weeks) post treatment; $98 \%$ of patients cleared C. difficile from their faeces at 4-8 weeks with no recurrences reported. Interestingly, the patient who received no prior antibiotic treatment also achieved a negative stool test result for $C$. difficile and showed improvement in symptoms after FMT treatment. No minor or serious adverse events were reported after the FMT treatments. Only one patient showed a positive stool test for $C$. difficile 5 weeks after treatment. However, this patient received antibiotics for treatment of Helicobacter pylori infection. The patient subsequently received further treatment with vancomycin followed by probiotics. Unfortunately, this patient was lost to followup.

There was a significant reduction in abdominal pain, diarrhoea, bloating and blood in the stool at 4-8 weeks post treatment (Fig. 1). Twenty-four patients had an extended 6 month follow-up of symptoms post treatment, which showed a significant improvement in abdominal pain, diarrhoea and blood in the stool from baseline $(P=0.004, P<0.001$ and $P=0.002)$. 


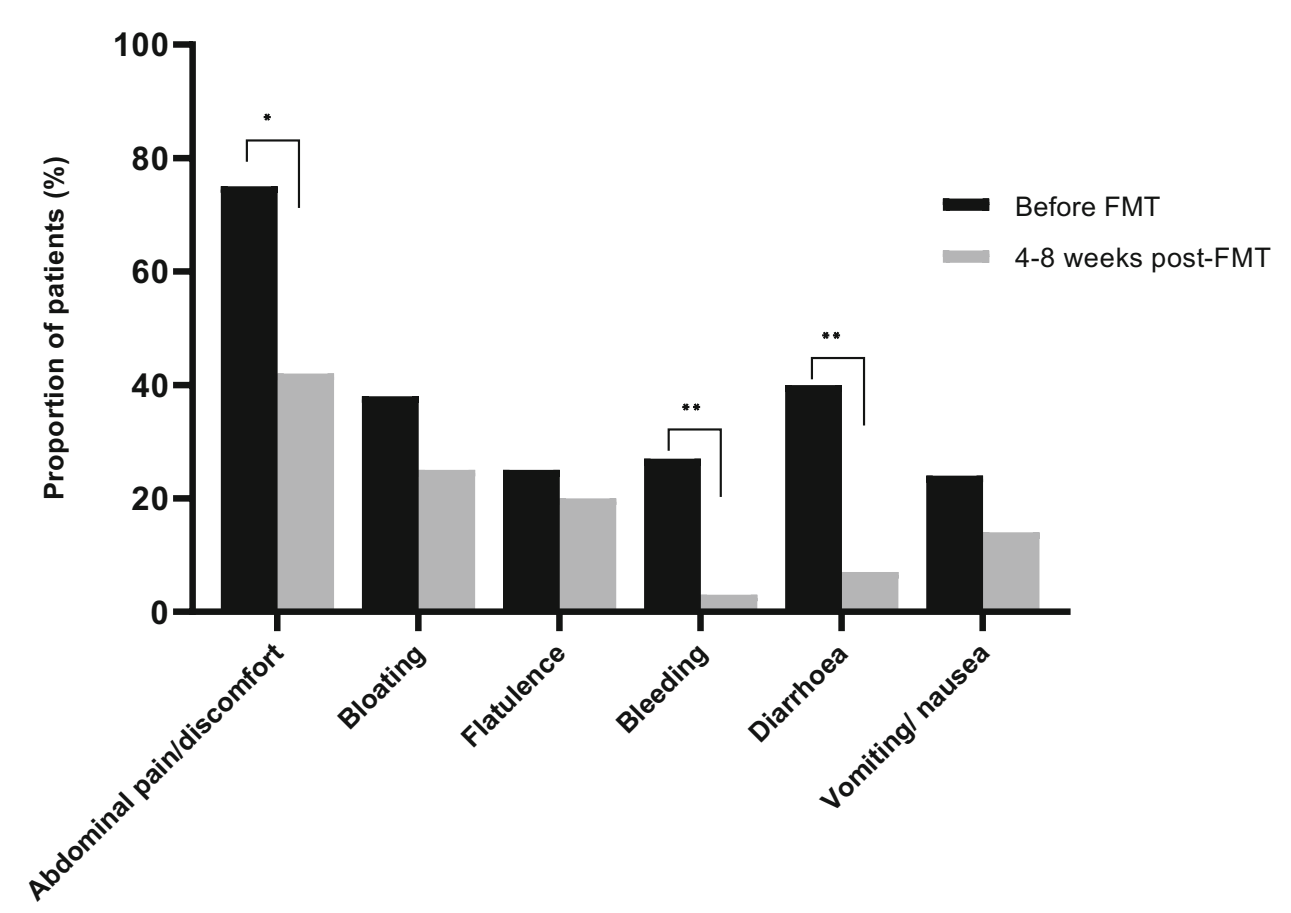

Fig. 1 Change in clinical symptoms pre and post treatment $(N=54)$. There was a significant reduction in symptoms of abdominal pain $(P=0.0035)$, rectal bleeding $(P=0.009)$ and diarrhoea $(P=0.0001)$ at the week $4-8$

\section{DISCUSSION}

This study demonstrates that FMT can be used safely and effectively as the first-line treatment for CDI. Overall, first-line treatment with FMT demonstrated high efficacy, low rates of recurrence, resolution of symptoms and no adverse events.

Globally, use of FMT and trials using FMT have increased markedly in the past 10 years. FMT is an emerging therapy for many indications, but there is strong evidence for its use in the treatment of CDI. However, currently it is mainly considered for treatment of recurrent CDI $[15,17]$. One clinical trial study has reported on 21 adult patients with acute CDI who had at least three loose stools per day, a positive C. difficile stool test and no previous CDI. Patients were treated with either metronidazole or FMT. The result showed an overall response of $78 \%$ in the FMT group compared to $45 \%$ in the metronidazole group. Although it was a smaller trial, the results suggested that FMT may be a follow-up post treatment. Statistical significance ${ }^{*} P<0.05,{ }^{* *} P<0.001$

better alternative to antibiotic therapy in the treatment of primary CDI [11]. Similarly, our study treated patients with a first occurrence of CDI, using two FMT infusions and achieved an efficacy of greater than $90 \%$. However, the main difference in our study was the use of pre-treatment antibiotics in all but one patient. Pretreatment antibiotics are used to manage the patient's symptoms whilst screening blood tests and preparation for FMT administration procedures are undertaken. However, as demonstrated by Juul et al. and the patient in our study, pre-treatment antibiotics may not be required [11]. A pooled analysis also showed no difference in FMT treatment outcome between patients receiving vancomycin pre-treatment and no pre-treatment groups [18]. This further supports the use of FMT as first-line treatment. It is time to optimize CDI treatment by focusing on FMT as first-line treatment. This will of course require prospective studies.

Treatment efficacy is known to be correlated with a number of other factors including the 
number of FMT infusions and route of delivery and thus these factors need to be considered when optimising treatment and clinical practice. One study has compared the cure rate among 80 immunocompromised patients with CDI treated with single versus multiple FMT. It showed a success rate of $88 \%$ after a single FMT whereas 93\% after multiple FMTs [19]. A systematic review in 2018 reported a cure rate of 93\% with multiple FMT and $76 \%$ after a single FMT [20]. In our study, all the participants received two consecutive FMT infusions and achieved a 98\% cure. Put together there is growing evidence supporting the use of a minimum of two FMT infusions for the treatment of CDI.

The route of delivery is another important factor in determining efficacy of FMT treatment. Cho et al. reported higher success rates for FMT delivered via colonoscopy (91\%) than via nasogastric or upper endoscopic route of delivery (82\%) [21]. Conversely, a pooled analysis study did not find any difference between the two methods. However, the sample size of patients receiving FMT as rectal enema was small $(n=34)$ unlike the colonoscopy group $(n=163)$ [18]. Another method for the delivery of faecal material is use of FMT capsules which is less invasive and more standardised. However, some microbes and metabolites that are critical for the efficacy of FMT may not survive the lyophilisation and encapsulation process or journey to the large bowel $[17,22]$. Therefore, it is necessary to determine the efficacy of fresh and processed faecal materials using different delivery methods.

This study provides clear information on the effectiveness of FMT as the initial treatment for CDI. There are limitations associated with our study, including the retrospective nature, lack of control group and a small sample size. Larger, prospective studies are required to confirm FMT as the first line of treatment for CDI.

\section{CONCLUSION}

Overall, this study demonstrates the efficacy and safety of FMT treatment for patients with a first episode of CDI. Our study supports the use of FMT as an optimal first-line treatment of CDI rather than waiting for multiple recurrences. Use of FMT as a first-line therapy could avoid further costs and consequences including increased mortality with delaying FMT. Further studies are required to confirm the effect of FMT for initial treatment of CDI and determine the pre-treatment, optimal delivery method and dose of FMT.

\section{ACKNOWLEDGEMENTS}

The authors would like to acknowledge the support of the Centre for Digestive Diseases nursing and research staff.

Funding. No funding or sponsorship was received for this study or publication of this article. The Rapid Service Fee was funded by the authors.

Authorship. All named authors meet the International Committee of Medical Journal Editors (ICMJE) criteria for authorship for this article, take responsibility for the integrity of the work as a whole, and have given their approval for this version to be published.

Prior Presentation. A subset of the data was presented as a poster at the 2015 American College of Gastroenterology Conference.

Disclosures. Niloufar Roshan and Annabel Clancy declare that they have no conflict of interest. Thomas Borody has a pecuniary interest in the Centre for Digestive Diseases, is a medical advisor to Finch Therapeutics and holds patents in FMT treatment.

Compliance with Ethics Guidelines. This study has been approved by the Centre for Digestive Diseases Human Research Ethics Committee (CDD19/C06). A waiver of consent was granted for this study. This study was performed in accordance with the Helsinki Declaration of 1964.

Data Availability. The datasets generated during and/or analyzed during the current 
study are not publicly available due to restriction under the ethics approval received for this study.

Open Access. This article is licensed under a Creative Commons Attribution-NonCommercial 4.0 International License, which permits any non-commercial use, sharing, adaptation, distribution and reproduction in any medium or format, as long as you give appropriate credit to the original author(s) and the source, provide a link to the Creative Commons licence, and indicate if changes were made. The images or other third party material in this article are included in the article's Creative Commons licence, unless indicated otherwise in a credit line to the material. If material is not included in the article's Creative Commons licence and your intended use is not permitted by statutory regulation or exceeds the permitted use, you will need to obtain permission directly from the copyright holder. To view a copy of this licence, visit http:// creativecommons.org/licenses/by-nc/4.0/.

\section{REFERENCES}

1. Clarkin C, Quist S, Shamis R, King A, Shah B. Management of Clostridioides difficile infection. Crit Care Nurse. 2019;39:e1-e13.

2. Riley TV. From obscurity to superbug; the rise of Clostridium difficile. Healthc Infect. 2010;15:59-61.

3. Spigaglia P. Recent advances in the understanding of antibiotic resistance in Clostridium difficile infection. Ther Adv Infect Dis. 2016;3(1):23-422.

4. Binion D. Clostridium difficile infection and inflammatory bowel disease. Gastroenterol Hepatol. 2016;13:334-7.

5. Eze P, Balsells E, Kyaw M, Nair H. Risk factors for Clostridium difficile infections: an overview of the evidence base and challenges in data synthesis. J Glob Health. 2017. https://doi.org/10.7189/jogh. 07.010417.

6. McDonald L, Gerding D, Johnson S, et al. Clinical practice guidelines for Clostridium difficile infection in adults and children: 2017 update by the Infectious Diseases Society of America (IDSA) and Society for Healthcare Epidemiology of America (SHEA). Clin Infect Dis. 2018;66:e1-48.

7. European Centre for Disease Prevention and Control. Healthcare-associated infections: Clostridium difficile infections. Annual epidemiological report for 2016. 2018. https://ecdc.europa.eu/sites/portal/ files/documents/AER_for_2016-C-difficile_0.pdf. Accessed 28 Aug 2020.

8. Hopkins R, Wilson R. Treatment of recurrent Clostridium difficile colitis: a narrative review. Gastroenterol Rep. 2018;6:21-8.

9. Lessa FC, Mu Y, Bamberg WM, et al. Burden of Clostridium difficile infection in the United States. N Engl J Med. 2015;372(9):825-34.

10. Bakken JS, Borody T, Brandt LJ, et al. Treating Clostridium difficile infection with fecal microbiota transplantation. Clin Gastroenterol Hepatol. 2011;9(12):1044-9.

11. Juul FE, Bretthauer $M$, Skudal $H$, et al. Faecal transplantation for primary Clostridium difficile infection. N Engl J Med. 2018;378:2535-6.

12. Jaworski A, Borody TJ, Leis S, Gadalla S, Dawson V. Treatment of first-time Clostridium difficile infection with fecal microbiota transplantation. Am J Gastroenterol. 2015;110(October):S587.

13. Borody TJ, Warren EF, Leis S, Surace R, Ashman O. Treatment of ulcerative colitis using fecal bacteriotherapy. J Clin Gastroenterol. 2003;37(1):42-7.

14. Borody TJ, Warren EF, Leis SM, Surace R, Ashman O, Siarakas S. Bacteriotherapy using fecal flora. J Clin Gastroenterol. 2004;38(6):475-83.

15. Cammarota G, Ianiro G, Tilg H, Al E. European consensus conference on FMT in clinical practice. BMJ Open. 2017;66:569-80.

16. Haifer C, Kelly CR, Paramsothy S, et al. Australian consensus statements for the regulation, production and use of faecal microbiota transplantation in clinical practice. Gut. 2020;69(5):801-10.

17. Wortelboer K, Nieuwdorp M, Herremaa H. Faecal microbiota transplantation beyond Clostridioides difficile infections. EBioMedicine. 2019;44:716-29.

18. Sofi A, Silverman A, Khuder S, Garborg K, Westerink J, Nawras A. Relationship of symptom duration and faecal bacteriotherapy in Clostridium difficile infection-pooled data analysis and a systematic review. Scand J Gastroenterol. 2013;48:266-73.

19. Kelly CR, Ihunnah C, Fischer M, et al. Fecal microbiota transplant for treatment of Clostridium 
difficile infection in immunocompromised patients. Am J Gastroenterol. 2014;109(7):1065-71.

20. Ianiro G, Masucci L, Quaranta G, et al. Randomised clinical trial: faecal microbiota transplantation by colonoscopy plus vancomycin for the treatment of severe refractory Clostridium difficile infection-single versus multiple infusions. Aliment Pharmacol Ther. 2018;48(2):152-9.
21. Cho J, Pestana L, Pardi R, Pardi D, Khanna S. Faecal microbiota transplant via colonoscopy may be preferred due to intraprocedure findings. Intest Res. 2019;17:434-7.

22. Kao D, Roach B, Silva M, et al. Effect of oral capsulevs colonoscopy-delivered fecal microbiota transplantation on recurrent Clostridium difficile infection. JAMA. 2017;318(20):1985. 